\title{
Programa de Controle da Doença de Chagas no Estado de São Paulo, Brasil: o controle e a vigilância da transmissão vetorial
}

\author{
Control Program of Chagas disease in São Paulo, Brazil: \\ the control and surveillance of vector transmission
}

\author{
Eduardo Olavo da Rocha e Silva*, Vera Lúcia Cortiço Corrêa Rodrigues*, \\ Rubens Antonio da Silva*e Dalva Marli Valério Wanderley*
}

\begin{abstract}
RESUMO
Discute-se o controle dos transmissores da doença de Chagas, no Estado de São Paulo, e as atividades que levaram à eliminação do Triatoma infestans. São destacados os fatores coadjuvantes as ações de controle, particularmente o êxodo rural. A partir de 1965, o combate tomou a forma de uma verdadeira campanha, com fases distintas em função das alterações epidemiológicas, experiência adquirida e pressão dos custos. Após 25 anos de trabalho a campanha foi considerada encerrada, com a eliminação dos focos da espécie do planalto paulista. Porém, em função da possibilidade da reintrodução de Triatoma iinfestans (transporte passivo) e da presença, em diversas localidades, de exemplares de espécies vetoras semidomiciliares (Triatoma sordida e Panstrogylus megistus) as atividades de controle não foram interrompidas. Em consequência, continuam em andamento as ações de vigilância entomológica.
\end{abstract}

Palavras-chaves: Doença de Chagas. Controle vetorial. Vigilância entomológica. Triatomíneo. Triatoma infestans.

\begin{abstract}
The control of the vectors of Chagas' disease in the State of Sao Paulo are discussed, mainly those activities that led to the elimination of Triatoma infestans. Secondary factors that helped the control such as rural exodus are also analyzed. The article shows that since 1965 the control became a campaign with different phases due to the epidemiological situation, the acquired knowledge and the entomological surveillance. After 25 years of work, the elimination of all the focus of Triatoma infestans was finally reached and the campaign was ended. However, due to the possibility of reintroduction of the vector in rural areas by passive transportation besides the presence of secondary vectors (Triatoma sordida and Panstrongylus megistus) in several localities, the vector control activities were not interrupted and the surveillance is continuous.
\end{abstract}

Key-words: Chagas disease. Vectorial control. Entomological surveillance. Triatominae. Triatoma infestans.

\section{INTRODUÇÃo}

Espécie alóctone, Triatoma infestans foi introduzido em terras paulistas, procedente do sul do país, provavelmente no decorrer do século XVIII, através dos tropeiros que percorriam o Caminho dos Muares, via esta que se alongava do Rio Grande do Sul até Sorocaba. Sua presença no Estado de São Paulo tomou vulto quando, no início do século passado, ocorreu maciço deslocamento da fronteira agrícola em direção ao oeste, em busca das terras virgens necessárias ao plantio do café ${ }^{1}$. Mas foi somente após a chegada dos europeus que essa dispersão se intensificou acompanhando a penetração e ocupação populacional, invadindo as habitações nos espaços abertos para atendimento das necessidades do plantio e criação de animais. Por possuir caráter mesotérmico, ou seja, preferência por ambientes áridos ou semiúmidos, T. infestans se adaptou bem aos espaços artificialmente abertos no planalto paulista, sobretudo, quando e onde predominavam as casas de pau-apique e/ou barro em péssimas condições de habitabilidade ${ }^{2}$.

* Superintendência de Controle de Endemias (SUCEN), Secretaria de Estado da Saúde, São Paulo, SP.

Endereço para correspondência: Drª Dalva Marli Valério Wanderley. SUCEN. Rua Paula Souza 166, 01027-000. São Paulo. SP.

Telefax: 5511 3311-1127

e-mail: dalva@sucen.sp.gov.br
Foi somente no decorrer da década dos anos quarenta que a problemática da doença de Chagas ganhou realmente força para buscar solução satisfatória. No Estado de São Paulo, o alarme levantado pela publicação de diversos trabalhos científicos ${ }^{3-6}$, mostrando a gravidade da situação então vigente, aliado a vontade política em recuperar a lavoura paulista da ruína financeira, consequente da queda do café no mercado internacional nos anos 30, foram os principais fatores que levaram o Governo e Assembléia estadual a delegar ao Serviço de Profilaxia da Malária (SPM) a responsabilidade de combater a endemia (lei estadual $n^{\circ}$ 1317 de 04/12/1951) 7 .

Tendo em vista seu grau de prioridade no âmbito do próprio serviço de controle das doenças transmitidas por vetores da Secretaria de Estado da Saúde, as ações contra Triatoma infestans desenvolveram-se em distintas fases: a) intermitente, durante o período de 1950 a 1959, caracterizada por atividades de controle não abrangentes nem contínuas; b) de trabalho intensificado, entre 1959 a 1964, realizado durante a Campanha de Erradicação da Malária (CEM) em áreas de sobreposição da presença de malária e doença de Chagas, visando o controle das duas endemias; c) a partir de 1964, com o término da fase mais ativa da CEM, ocorreu à atividade denominada arrastão, caracterizada pela aplicação de inseticidas (benzene hexachloride-BHC 30\%) em todas as casas e seus anexos da 
zona rural; d) de expurgo seletivo, de 1968 a 1972, com pesquisa em casas e anexos, situados em áreas da zona rural; expurgo naquelas onde se evidenciara a presença de triatomíneos ${ }^{7-9}$, de acordo com metodologia preconizada por Freitas ${ }^{10}$; e) prioridades, de 1973 a 1983, onde se adotou o conceito de estratificação epidemiológica (prioridades) envolvendo medidas estruturadas com base no risco da transmissão da doença, adequando a periodicidade da pesquisa de triatomíneos aos índices de infestação de cada espécie de destacada importância epidemiológica'; f) consolidação/vigilância entomológica (1984 aos dias atuais) eliminação dos focos residuais de Triatoma infestans e implantação da vigilância entomológica para espécies secundárias.

No presente artigo, são apresentadas as ações de controle vetorial empreendidas sobre a principal espécie, T. infestans e os resultados atuais da vigilância entomológica instituída, discutindo-se as perspectivas dessa estratégia no estado.

\section{MÉTODOS}

São descritos os resultados obtidos no Programa de Controle da Doença de Chagas no Estado de São Paulo, pela Superintendência de Controle de Endemias (SUCEN) e no passado pelas instituições que a antecederam, desde 1950, segundo as fases consideradas. A fonte de dados inclui relatórios internos, sistema de informações, artigos, monografias e teses de técnicos do serviço.

Foi analisada a série histórica de presença de triatomíneos segundo espécie e positividade para tripanossomatídeos, casas e anexos pesquisados e positivos para triatomíneos, distribuição geográfica da principal espécie, Triatoma infestans, em anos que constituem marcos nas diversas fases do programa, destacando-se o recurso destinado à campanha em cada fase, segundo a literatura e convertidos a preços da moeda atual, atualizado segundo Índice Geral de Preços da Fundação Getúlio Vargas. Para o cálculo dos custos das medidas de vigilância entomológica, foram apurados os itens que compõem a atividade de pesquisa e borrifação e calculados, para cada um deles, os valores anuais em moeda brasileira (real-R\$). Para a conversão dos valores em reais para a moeda americana (dólar-US\$) foi utilizado como referência o mês e ano da tomada de preço de cada item, ou seja, maio/2008. Foram considerados os componentes de ordem operacional tais como: veículos, pulverizadores, insumos estratégicos (inseticida), equipamento de proteção individual (EPI), uniforme, combustível, material para pesquisa entomológica, mostruários e impressos, além de despesas com pessoal/ reflexos e diárias. Foram computados os custos de investimento para a estruturação de equipes regionalizadas em 22 setores do estado, com atribuições de operações de campo, educação e supervisão, referentes a veículos e pulverizadores, ao lado das despesas de custeio, como a depreciação no ano (considerando vida útil de 10 anos) e manutenção, somados aos ítens acima.

Os custos utilizados com recursos humanos foram calculados a partir do salário atual e os encargos a ele atribuído, considerando-se o rendimento de uma unidade domiciliar/ homem/dia para o atendimento de notificação com borrifação e 3 unidades domiciliares/homem/dia para atendimento de notificação negativa, portanto, sem borrifação. Desta forma, foram considerados para a equipe de campo, composta por um desinsetizador, um encarregado e um motorista a destinação de $30 \%$ da jornada anual para este trabalho. Para as equipes de educação, formadas por um visitador sanitário e um motorista, e para as equipes de supervisão formadas por um encarregado e um motorista foram consideradas a dedicação de $25 \%$ da jornada de trabalho anual de 210 dias úteis.

$\mathrm{Na}$ fase atual, são apresentadas informações referentes à metodologia da vigilância instituída por meio de notificações de triatomíneos espontâneas pela população.

\section{RESULTADOS}

\section{Fase intermitente, período de 1950 a 1959}

No início das atividades de controle dos transmissores da doença de Chagas, a região endêmica era vista como uma área ampla e contínua, atingindo todo o planalto paulista, compreendendo 219 municípios, dos quais em 147 foi constatada a presença de T. infestans infectado por Trypanosoma cruzi $^{7-9}$, distribuição esta bastante ampliada quando comparada à primeira realizada em 1916 (Figura 1), com expansão da infestação para o oeste do estado nas décadas de 1920 a $1950^{7}$. As operações de campo perfizeram 2.481.494 casas tratadas entre os anos de 1951 a 1959, ou seja, uma média de 275.722 casas/ano, significando uma cobertura/ano de $34,5 \%$ da estimativa de 800.000 casas existentes na região endêmica. O não alcance da totalidade das casas e, em certos casos, nem mesmo todas as localidades deveu-se, a limitação orçamentária, à inexistência de mapas (croquis de localidades) e dificuldades no deslocamento (deficiência no transporte). Dado que o então Serviço de Profilaxia da Malária incorporou a ação contra os triatomíneos, e uma vez que havia semelhança técnica entre os métodos de aplicação de DDT e BHC, utilizando o mesmo equipamento, apenas com modificação no bico de aspersão para, ao invés do jato em leque, se obter um jato mais fechado com maior penetração nas frestas das paredes, onde se alojavam os triatomíneos, a preparação do pessoal foi rápida. A comparação das áreas malárica e triatomínica permitia separar regiões onde os trabalhos seriam feitos conjuntamente ou separados. Nas áreas de superposição, a mesma bomba aspersora aplicava mistura de BHC e DDT capaz de deixar um resíduo na parede de $0,25 \mathrm{~g} / \mathrm{m}^{2}$, respeitando a prioridade das áreas onde a malária se apresentava de forma epidêmica. o trabalho foi possível em virtude de o serviço contar com equipes sediadas nas diversas regiões administrativas do estado, cada uma responsável por um determinado número de domicílios, situação que permanece até hoje. Os triatomíneos capturados eram analisados quanto à espécie e infecção natural e amostras de sangue da população residente eram examinadas para avaliação da prevalência. Um dos alvos ou objetivos, na ocasião, era baixar o número de triatomíneos nas casas para diminuir a ocorrência da transmissão, fato este que pode ser constatado. Enquanto a média anual de T. infestans capturados para os 5 primeiros anos do período foi de 39.712 exemplares, para os 5 últimos a média obtida foi de 19.040 exemplares $^{7}$. 


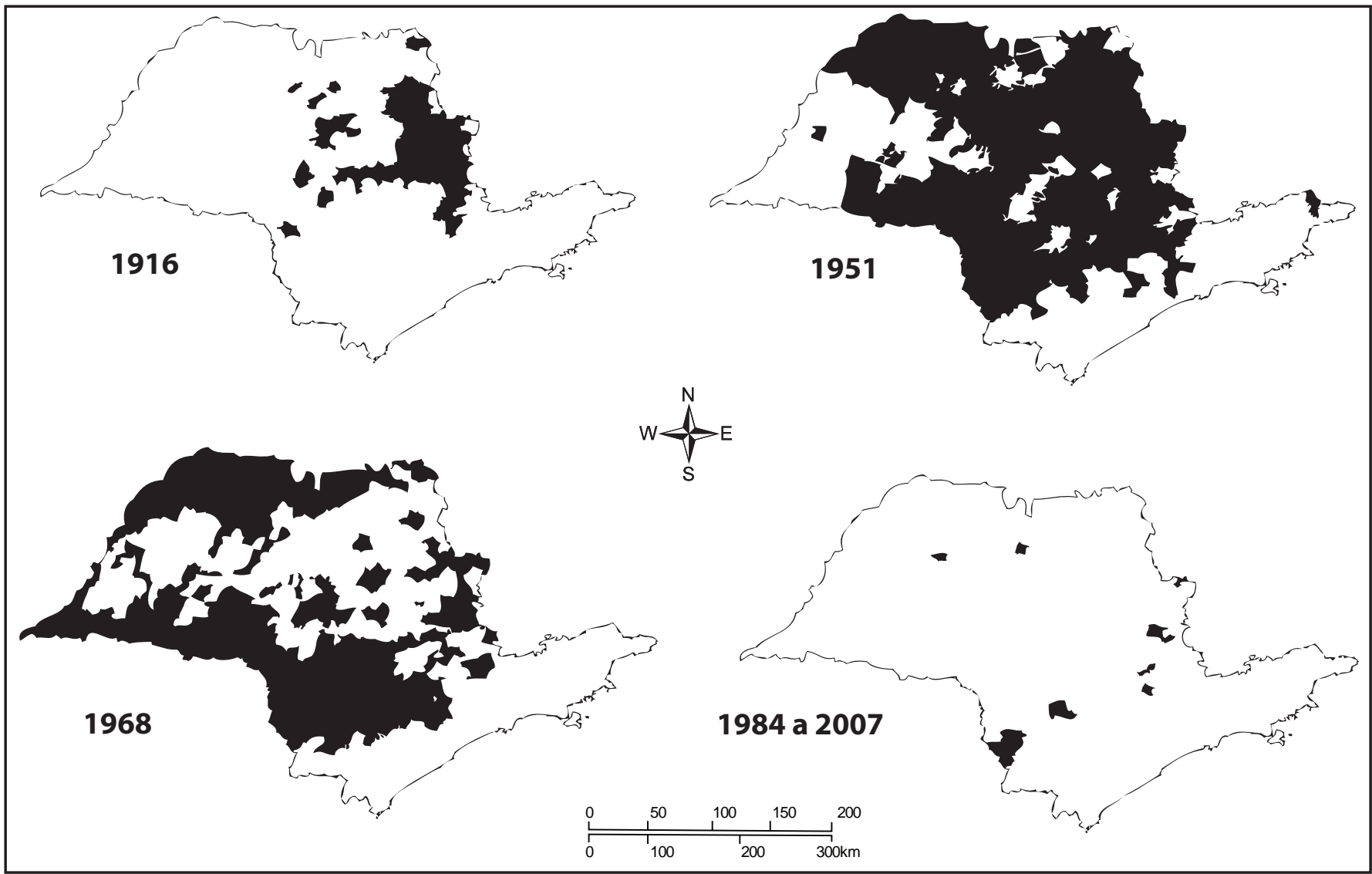

FIGURA 1 - Presença de Triatoma infestans em Municípios do Estado de São Paulo.

No entanto, decorridos nove anos de início do controle a situação continuava grave. O índice de infecção natural mostrava-se elevado e o resultado dos exames sorológicos, a despeito da pouca representatividade das amostras coletadas, embora apresentando decréscimo no período encontrava-se no patamar de $9 \%{ }^{7}$. Em 1959, Corrêa e Ferreira ${ }^{11}$ examinaram 86.992 exemplares de T. infestans e encontraram um índice geral de infecção de 9,4\%. A dotação orçamentária anual para esta fase, calculada sobre valores para o período de 1951 a 1954 foi de $\operatorname{Cr} \$ 5.382 .127,25^{7,8}$, ou seja, a preços de hoje, $\mathrm{R} \$ 3.149 .000,00$, ou US $\$ 1.933 .000,00$.

Fase de trabalho intensificado, entre 1959 a 1964

Inicialmente intermitente, as ações contra T. infestans foram incrementadas a partir de 1959 até 1964, durante a Campanha de Erradicação da Malária (CEM), com o trabalho intensificado em áreas de sobreposição da presença da malária e doença de Chagas, visando ao controle das duas endemias, porém a campanha de Chagas sofreu injunções decorrentes da maior prioridade dada à luta contra a malária, ou seja, manteve o T. infestans em situação estacionária, permitindo o avanço do T. sordida ${ }^{9}$. Neste período, o SPM se transformou em Serviço de Erradicação da Malária e Profilaxia da Doença de Chagas (SEMPDC). Inquérito de soroprevalência para infecção chagásica realizado no início desta fase apontou 9,3\% de positividade na população geral e 6,3\% em indivíduos com idades inferiores a 9 anos, indicando transmissão vetorial recente e ativa ${ }^{12}$. Ao mesmo tempo, o aumento do número de exemplares das espécies secundárias Triatoma sordida e Panstrongylus megistus constituíam motivo de inquietação $0^{7-9}$.
Durante esta fase, foi concluída a etapa mais ativa da Campanha de Erradicação da Malária no Estado, o que trouxe alívio ao orçamento do Serviço além da ampliação do recurso humano para as ações de controle da Doença de Chagas. Neste período, o programa apresentou média anual das despesas de Cr\$ 5.542.066,75, calculados para o período de 1961 a $1964^{8}$, valor este que, na moeda brasileira atual, corresponde a $\mathrm{R} \$ 2.570 .000,00$ e US $\$ 1.577 .000,00$.

\section{Fase do arrastão}

Sem dúvida, a experiência adquirida em programação, execução e supervisão das operações de campo pelos técnicos do serviço contribuiu para o desencadeamento de uma ampla ação de ataque efetivo aos vetores, chamado Arrastão, aliado ao aporte maior de recursos. No período de 1965 a 1967, foram desinsetizadas todas as casas da área endêmica para eliminar os barbeiros, em ciclos anuais. A desinsetização foi realizada com o emprego do BHC 30\%, pó molhável, numa dose média aproximada de 0,5 gramas por metro quadrado de superfície tratada. A borrifação seguia um ritmo mais lento do que o adotado para a malária, para que a suspensão pudesse penetrar mais fundo nas fendas e rachaduras existentes nas superfícies tratadas.

Devido ao tempo gasto na operação de desinsetização, a atividade de pesquisa e captura de triatomíneos era realizada em apenas uma de cada três casas trabalhadas. Nas casas não selecionadas para pesquisa, após o tratamento o desinsetizador observava nos cômodos trabalhados a presença de eventuais triatomíneos intoxicados na superfície das paredes tratadas, ou caídos no chão e os recolhia. 
Em que pese a não realização da pesquisa de forma integral, em todas as casas, porém em apenas 30\% delas, foram capturados, nos três anos da etapa do arrastão, 279.082 exemplares de T. infestans, dos quais 174.534 foram examinados, com um índice de infecção por T. cruzi de $2 \%$. Constatou-se também que a distribuição de T. infestans já não apresentava a continuidade anotada na década anterior e simuma distribuição descontínua (mosaico). A nova distribuição da espécie (Figura 1), o elevado custo da operação, a poluição ambiental pelo uso de inseticida e outros fatores aconselhavam uma mudança de estratégia. Desta forma, foi introduzido o método seletivo ${ }^{13}$, conforme preconizado por Pedreira de Freitas ${ }^{10}$.

0 custo médio nos três anos do arrastão atingiu a cifra de $\mathrm{CR} \$ 69.631 .962,66^{8}$, ou seja, $\mathrm{R} \$ 32.289 .000,00$ na moeda brasileira atual e US\$19.820.000,00.

\section{Fase de expurgo seletivo, período de 1968 a 1972}

Nesta etapa, após uma avaliação da área de distribuição dos triatomíneos, o Serviço de Erradicação da Malária e Profilaxia da Doença de Chagas (SEMPDC) passou a dar prioridade à atividade de pesquisa cuidadosa do vetor em todas as casas e anexos rurais da área endêmica, com a utilização do desalojante (Piriza a 1\%), e a realizar expurgo somente nas Unidades Domiciliares (UDs) infestadas. Com relação à extensão do trabalho realizado, no primeiro ano do seletivo, o número de casas pesquisadas foi de 884.812 e de anexos 1.485.299 (Tabela 1). Se for considerado que no período de 1960 a 1970 ocorreu uma redução de $26,9 \%$ da população rural e, consequentemente do número de habitações $(29,1 \%)$ e que pelo censo de 1970 havia 657.787 casas na zona rural (Tabela 2), é de se supor que a área pesquisada abrangeu toda e qualquer habitação rural e periurbana do território paulista, o que permitiu demarcar exatamente a situação da infestação do Estado (Figura 1).
TABELA 1 - Casas e anexos/peridomicílios pesquisados e positivos em rotina de pesquisa de triatomíneos. Estado de São Paulo, 1968 a 2001.

\begin{tabular}{|c|c|c|c|c|c|c|c|}
\hline \multirow[b]{2}{*}{ Fase } & \multirow[b]{2}{*}{ Ano } & \multicolumn{3}{|c|}{ Casas } & \multicolumn{3}{|c|}{ Anexos/peridomicílios } \\
\hline & & pesquisadas & infestadas & $\%$ & pesquisados & infestados & $\%$ \\
\hline \multirow{5}{*}{ Seletivo } & 1968 & 884.812 & 18.556 & 2,1 & 1.485 .299 & 34.389 & 2,3 \\
\hline & 1969 & 733.109 & 17.143 & 2,3 & 1.391 .532 & 36.743 & 2,6 \\
\hline & 1970 & 639.423 & 11.585 & 1,8 & 1.060 .693 & 32.907 & 3,1 \\
\hline & 1971 & 656.796 & 9.478 & 1,4 & 1.083 .822 & 35.314 & 3,3 \\
\hline & 1972 & 572.635 & 5.828 & 1,0 & 1.138 .891 & 24.674 & 2,2 \\
\hline \multirow{11}{*}{ Prioridades } & 1973 & 562.164 & 3.752 & 0,7 & 1.082 .932 & 17.625 & 1,6 \\
\hline & 1974 & 488.311 & 2.456 & 0,5 & 964.911 & 15.984 & 1,7 \\
\hline & 1975 & 457.290 & 2.187 & 0,5 & 918.014 & 17.051 & 1,9 \\
\hline & 1976 & 445.916 & 1.752 & 0,4 & 900.047 & 13.720 & 1,5 \\
\hline & 1977 & 417.909 & 1.162 & 0,3 & 844.747 & 9.661 & 1,1 \\
\hline & 1978 & 459.836 & 1.076 & 0,2 & 888.457 & 9.079 & 1,0 \\
\hline & 1979 & 430.782 & 760 & 0,2 & 855.991 & 6.700 & 0,8 \\
\hline & 1980 & 437.250 & 711 & 0,2 & 846.317 & 6.081 & 0,7 \\
\hline & 1981 & 438.079 & 808 & 0,2 & 888.442 & 8.860 & 1,0 \\
\hline & 1982 & 370.070 & 638 & 0,2 & 782.558 & 7.465 & 1,0 \\
\hline & 1983 & 366.627 & 532 & 0,1 & 741.897 & 6.109 & 0,8 \\
\hline \multirow{18}{*}{ Vigilância } & 1984 & 278.686 & 298 & 0,1 & 517.932 & 3.707 & 0,7 \\
\hline & 1985 & 237.993 & 808 & 0,3 & 185.542 & 3.048 & 2,0 \\
\hline & 1986 & 184.185 & 650 & 0,4 & 142.787 & 2.720 & 2,0 \\
\hline & 1987 & 159.186 & 671 & 0,2 & 126.589 & 2.003 & 2,0 \\
\hline & 1988 & 74.816 & 60 & 0,08 & 61.692 & 306 & 0,5 \\
\hline & 1989 & 60.217 & 86 & 0,1 & 51.880 & 1.339 & 3,0 \\
\hline & 1990 & 12.142 & 95 & 0,8 & 10.339 & 767 & 7,4 \\
\hline & 1991 & 13.462 & 124 & 0,9 & 12.145 & 873 & 7,2 \\
\hline & 1992 & 21.038 & 206 & 0,9 & 18.911 & 1.394 & 7,3 \\
\hline & 1993 & 21.931 & 278 & 1,2 & 19.194 & 1.551 & 8,1 \\
\hline & 1994 & 19.858 & 349 & 1,7 & 17.833 & 1.777 & 9,9 \\
\hline & 1995 & 13.314 & 235 & 1,8 & 12.461 & 1.615 & 12,9 \\
\hline & 1996 & 8.349 & 185 & 2,2 & 7.332 & 973 & 13,3 \\
\hline & 1997 & 17.314 & 388 & 2,2 & 15.661 & 1.574 & 10,0 \\
\hline & 1998 & 12.015 & 286 & 2,4 & 10.732 & 976 & 9,1 \\
\hline & 1999 & 12.590 & 277 & 2,2 & 10.480 & 920 & 8,8 \\
\hline & 2000 & 9.874 & 368 & 3,7 & 8.781 & 963 & 10,9 \\
\hline & 2001 & 8.237 & 303 & 3,4 & 7.371 & 660 & 8,9 \\
\hline Total & & 9.526 .216 & 84.091 & 0,9 & 17.112 .212 & 309.528 & 1,8 \\
\hline
\end{tabular}

Deve ser considerado que o modelo operacional e logístico utilizado, no decorrer das fases iniciais do controle da doença de Chagas, foi aquele seguido pela Campanha de Erradicação da Malária, adaptado às especificidades do combate a uma nova endemia.

Registre-se que durante esta fase houve reorganização do SEMPDC que se transformou numa Autarquia SUSAM (Superintendência do Saneamento Ambiental), responsável pela Campanha de Erradicação da Malária, Controle da Doença de Chagas e da Poluição do Ar. Em 1975, uma nova re-estruturação transferiu as atribuições de controle da poluição do ar para a CETESB e a SUSAM passou a denominar-se SUCENSuperintendência de Controle de Endemias.

No final da fase, foi constatada uma significativa redução no número de exemplares de T. infestans capturados, de 69.229, em 1968, para 6.046 exemplares, em 1972 (Tabela 3). No entanto, a diminuição dos municípios infestados não foi significativa, de 182 municípios, em 1968, para 159, em 1972. De qualquer maneira, ficara claro ser possível eliminar a espécie de determinadas áreas.

Quanto à infestação das casas, foi verificada queda acentuada, com variação segundo regiões do estado, destacando as de Sorocaba, RibeirãoPreto e São José do Rio Preto com os maiores percentuais, quando comparados com a média do estado, não se observando o mesmo comportamento quando considerada a infestação dos anexos ${ }^{7}$. 
TABELA 2 - População rural e domicílios rurais segundo ano censitário no Estado de São Paulo.

\begin{tabular}{|c|c|c|c|c|}
\hline \multirow[b]{2}{*}{ Ano } & \multicolumn{2}{|c|}{ População rural } & \multicolumn{2}{|c|}{ Domicílios rurais } \\
\hline & $\mathrm{n}^{\circ}$ & \% variação & $\mathrm{n}^{\circ}$ & \% variação \\
\hline 1950 & 4.330 .212 & & 824.396 & \\
\hline 1960 & 4.824 .720 & 11,4 & 927.787 & 12,5 \\
\hline 1970 & 3.526 .499 & $-26,9$ & 657.787 & $-29,1$ \\
\hline 1980 & 2.844 .334 & $-19,4$ & 588.103 & $-10,6$ \\
\hline 1991 & 2.275 .068 & $-20,1$ & 559.576 & $-4,8$ \\
\hline 2000 & 2.436 .374 & 7,0 & 673.699 & 20,3 \\
\hline
\end{tabular}

TABELA 3 - Triatomíneos coletados, examinados e positivos, segundo espécie. Estado de São Paulo, 1968 a 2007.

\begin{tabular}{|c|c|c|c|c|c|c|c|c|c|c|c|c|c|}
\hline \multirow[t]{2}{*}{ Fase } & \multirow[b]{2}{*}{ Ano } & \multicolumn{3}{|c|}{ Triatoma infestans } & \multirow[b]{2}{*}{$\%$} & \multicolumn{3}{|c|}{ Triatoma sordida } & \multicolumn{5}{|c|}{ Panstrongylus megistus } \\
\hline & & coletados & examinados & positivos & & coletados & examinados & positivos & $\%$ & coletados & examinados & positivos & $\%$ \\
\hline \multirow{5}{*}{ Seletivo } & 1968 & 69.229 & 51.744 & 2.020 & 3,9 & 114.118 & 65.161 & 282 & 0,4 & 10.582 & 8.075 & 54 & 0,7 \\
\hline & 1969 & 48.126 & 40.172 & 904 & 2,3 & 88.880 & 46.514 & 305 & 0,7 & 9.535 & 8.122 & 16 & 0,2 \\
\hline & 1970 & 26.641 & 19.678 & 309 & 1,6 & 63.225 & 41.905 & 419 & 1,0 & 6.281 & 4.305 & 68 & 1,6 \\
\hline & 1971 & 12.374 & 10.656 & 311 & 2,9 & 55.459 & 36.684 & 562 & 1,5 & 3.390 & 2.852 & 31 & 1,1 \\
\hline & 1972 & 6.046 & 5.133 & 90 & 1,8 & 46.206 & 35.228 & 432 & 1,2 & 5.247 & 4.632 & 293 & 6,3 \\
\hline \multirow{11}{*}{ Prioridades } & 1973 & 4.081 & 3.249 & 39 & 1,2 & 37.579 & 27.331 & 209 & 0,8 & 5.839 & 4.655 & 259 & 5,6 \\
\hline & 1974 & 2.313 & 1.906 & 14 & 0,7 & 33.285 & 26.406 & 187 & 0,7 & 9.052 & 6.224 & 331 & 5,3 \\
\hline & 1975 & 713 & 575 & 3 & 0,5 & 34.277 & 26.745 & 183 & 0,7 & 4.069 & 3.534 & 349 & 9,9 \\
\hline & 1976 & 540 & 444 & 1 & 0,2 & 28.284 & 21.917 & 77 & 0,4 & 4.662 & 4.192 & 191 & 4,6 \\
\hline & 1977 & 370 & 297 & 0 & 0,0 & 19.551 & 16.165 & 81 & 0,5 & 3.992 & 3.251 & 262 & 8,1 \\
\hline & 1978 & 1.227 & 1.123 & 5 & 0,4 & 19.048 & 15.005 & 41 & 0,3 & 3.518 & 3.034 & 313 & 10,3 \\
\hline & 1979 & 104 & 89 & 0 & 0,0 & 14.862 & 12.973 & 16 & 0,1 & 4.734 & 3.695 & 222 & 6,0 \\
\hline & 1980 & 32 & 30 & 0 & 0,0 & 16.230 & 14.133 & 5 & 0,0 & 2.782 & 2.370 & 613 & 25,9 \\
\hline & 1981 & 46 & 46 & 0 & 0,0 & 15.523 & 15.523 & 14 & 0,1 & 1.976 & 1.976 & 195 & 9,9 \\
\hline & 1982 & 83 & 83 & 0 & 0,0 & 20.880 & 20.880 & 42 & 0,2 & 2.726 & 2.726 & 131 & 4,8 \\
\hline & 1983 & 25 & 25 & 0 & 0,0 & 15.966 & 15.966 & 46 & 0,3 & 4.345 & 4.345 & 689 & 15,9 \\
\hline \multirow{24}{*}{ Vigilância } & 1984 & 142 & 140 & 0 & 0,0 & 16.118 & 9.697 & 34 & 0,4 & 2.931 & 2.418 & 47 & 1,9 \\
\hline & 1985 & 2 & 2 & 0 & 0,0 & 20.719 & 17.165 & 63 & 0,4 & 4.298 & 3.913 & 353 & 9,0 \\
\hline & 1986 & 1 & 1 & 0 & 0,0 & 14.698 & 12.297 & 61 & 0,5 & 2.410 & 1.900 & 104 & 5,5 \\
\hline & 1987 & 1 & 1 & 0 & 0,0 & 12.297 & 10.547 & 137 & 1,3 & 3.290 & 2.948 & 194 & 6,6 \\
\hline & 1988 & 10 & 10 & 0 & 0,0 & 7.911 & 7.114 & 78 & 1,1 & 1.920 & 1.611 & 280 & 17,4 \\
\hline & 1989 & 39 & 39 & 0 & 0,0 & 9.772 & 9.253 & 57 & 0,6 & 2.063 & 1.842 & 134 & 7,3 \\
\hline & 1990 & 1 & 1 & 0 & 0,0 & 7.013 & 6.133 & 19 & 0,3 & 1.643 & 1.572 & 137 & 8,7 \\
\hline & 1991 & 0 & 0 & 0 & 0,0 & 6.169 & 5.544 & 19 & 0,3 & 1.519 & 1.351 & 51 & 3,8 \\
\hline & 1992 & 0 & 0 & 0 & 0,0 & 8.742 & 7.654 & 19 & 0,2 & 2.253 & 1.982 & 43 & 2,2 \\
\hline & 1993 & 0 & 0 & 0 & 0,0 & 8.280 & 7.490 & 64 & 0,9 & 1.428 & 1.129 & 133 & 11,8 \\
\hline & 1994 & 1 & 1 & 0 & 0,0 & 9.810 & 8.476 & 47 & 0,6 & 1.514 & 1.453 & 238 & 16,4 \\
\hline & 1995 & 0 & 0 & 0 & 0,0 & 6.874 & 6.236 & 39 & 0,6 & 503 & 403 & 32 & 7,9 \\
\hline & 1996 & 0 & 0 & 0 & 0,0 & 8.712 & 7.971 & 34 & 0,4 & 659 & 545 & 47 & 8,6 \\
\hline & 1997 & 0 & 0 & 0 & 0,0 & 11.569 & 10.634 & 106 & 1,0 & 845 & 803 & 21 & 2,6 \\
\hline & 1998 & 2 & 2 & 0 & 0,0 & 6.358 & 5.174 & 22 & 0,4 & 512 & 416 & 7 & 1,7 \\
\hline & 1999 & 106 & 35 & 0 & 0,0 & 5.845 & 4.229 & 32 & 0,8 & 635 & 510 & 9 & 1,8 \\
\hline & 2000 & 0 & 0 & 0 & 0,0 & 8.172 & 7.265 & 34 & 0,5 & 122 & 93 & 6 & 6,5 \\
\hline & 2001 & 0 & 0 & 0 & 0,0 & 7.332 & 6.469 & 18 & 0,3 & 371 & 326 & 34 & 10,4 \\
\hline & 2002 & 0 & 0 & 0 & 0,0 & 7.401 & 6.760 & 12 & 0,2 & 538 & 456 & 61 & 13,4 \\
\hline & 2003 & 0 & 0 & 0 & 0,0 & 5.227 & 4.709 & 16 & 0,3 & 450 & 430 & 25 & 5,8 \\
\hline & 2004 & 0 & 0 & 0 & 0,0 & 7.494 & 7.121 & 14 & 0,2 & 807 & 724 & 49 & 6,8 \\
\hline & 2005 & 0 & 0 & 0 & 0,0 & 14.564 & 13.993 & 23 & 0,2 & 839 & 735 & 52 & 7,1 \\
\hline & 2006 & 0 & 0 & 0 & 0,0 & 9.031 & 8.547 & 22 & 0,3 & 715 & 598 & 86 & 14,4 \\
\hline & 2007 & 0 & 0 & 0 & 0,0 & 6.238 & 5.922 & 11 & 0,2 & 439 & 409 & 44 & 10,7 \\
\hline Total & & 172.255 & 135.482 & 3.696 & 2,7 & 849.719 & 634.936 & 3.882 & 0,6 & 115.434 & 96.555 & 6.204 & 6,4 \\
\hline
\end{tabular}


No que diz respeito às despesas com o programa, nesta fase, pode-se constatar um custo médio anual de $\mathrm{Cr} \$ 37.084 .446,12$ a preço de $1978^{8}$, significando na moeda brasileira atual $\mathrm{R} \$ 17.196 .000,00$ e US $\$ 10.600 .000,00$.

\section{Fase de prioridades, período de 1973 a 1983}

A partir de 1973, adotou-se o conceito de estratificação epidemiológica (prioridades) envolvendo medidas estruturadas, adequando-se as exigências de redução das despesas e a periodicidade da pesquisa de triatomíneos aos índices de infestação de cada espécie de destacada importância epidemiológica. Além de Triatoma infestans, as espécies que serviram de base para as avaliações foram Triatoma sordida e Panstrongylus megistus. Em municípios com a presença do Triatoma infestans (Prioridade 1), pesquisas de todas as casas e anexos foram realizadas anualmente, com revisão de focos, isto é, nova pesquisa nas casas e anexos borrifados, 60 a 120 dias após a borrifação. Aqueles com presença de T. sordida e P. megistus (Prioridade 2), seriam trabalhados a cada dois anos, enquanto que, nos municípios sem grandes problemas de invasão de outras espécies (Prioridade 3), a rigor, em fase de vigilância entomológica, as atividades de pesquisa programada seriam suspensas. Na prática, esses municípios em Prioridade 3 permaneceram pesquisados com periodicidade trienal. A situação de cada município era avaliada anualmente. Caso fosse viável uma mudança de prioridade, a documentação do município em questão era encaminhada para uma Comissão de Avaliação designada especialmente para realizar essa tarefa. Com base nos dados encaminhados, era autorizada ou não a mudança solicitada ${ }^{9}$. Nesta fase, foram sendo instalados sistemas de recebimento de notificações encaminhadas pela população e o pronto atendimento.

o acompanhamento dos indicadores entomológicos para o período demonstrou diminuição do número de casas pesquisadas e infestadas por triatomíneos (Tabela 1), assim como do encontro de exemplares de T. infestans, $P$. megistus e T. sordida. O sucesso do controle dos transmissores da doença por meio químico foi aliado ao gradativo esvaziamento demográfico rural (Tabela 2), à demolição de habitações de má qualidade e à melhora do nível econômico da população do meio rural ${ }^{8,9}$. O encontro dos últimos exemplares de T. infestans infectados por T. cruzi no estado ocorreram no ano de 1978 (Tabela 3) em 4 municípios da região de Sorocaba. Neste ano, o número de municípios com T.infestans apresentava um decréscimo de 51,6\% em relação a 1973, ano do início da fase das prioridades ${ }^{7}$.

A Campanha destinada a eliminar os focos de T. infestans teve como objetivo acabar com as colônias deste triatomíneo no planalto paulista e foi sendo consolidada aos poucos, exigindo diferentes etapas ou táticas de trabalho que ocuparam um longo tempo (25 anos) para chegar à meta final. Porém, isto somente se tornou possível devido a uma somatória de fatores de difícil ponderação, mas de fácil indicação: aloctonia e hábito domiciliar da espécie (fatores endógenos); êxodo rural e demolição das casas com precárias condições de habitabilidade (fatores populacionais); desmatamento, de importância na redução das espécies secundárias (fator físico); experiência dos responsáveis pela campanha (fator técnico) e dotação orçamentária e pessoal habilitado suficiente (fatores econômicos) ${ }^{8,9}$.
Esta fase teve um custo médio anual de $\mathrm{Cr} \$ 47.969 .966,00$ a preço de $1978^{8}$, ou seja, $\mathrm{R} \$ 22.000 .000,00$ na moeda brasileira atual e US\$13.502.000,00.

Fase de consolidação/vigilância entomológica (1984 aos dias atuais)

No início desteperíodo, a avaliação do programa demonstrava que a transmissão natural por triatomíneos domiciliados estava interrompida; a presença de $T$. infestans estava restrita a focos residuais isolados, sem tendência de crescimento da infestação; Triatoma sordida presente em área geográfica extensa, porém com baixos índices de infecção por T. cruzi e com tendência de decréscimo; Panstrongylus megistus distribuído em área mais restrita que T. sordida, porém apresentando focos com elevado número de exemplares infectados colonizando, preferencialmente, anexos e casas desabitadas.

o Programa passou a ter como objetivo geral manter a interrupção da transmissão natural da doença de Chagas e, como objetivos específicos: investigar a sororreatividade em populações humanas sujeitas ao contato com triatomíneos infectados, encaminhando para atendimento no sistema de saúde os portadores da infecção chagásica; identificar e combater focos domiciliares ${ }^{14}$ contribuindo para a redução das fontes de infecção. Foram propostas ações relacionadas com o controle da infestação da Unidade Domiciliar (UD) por triatomíneos (programação de pesquisa de rotina em todas as UDs de localidades infestadas; recebimento e atendimento imediato de notificações feitas pela população e tratamento com inseticida da UD com presença de focos de triatomíneos) ${ }^{15}$. Novos critérios foram estabelecidos para a classificação das prioridades, baseados nos resultados obtidos nos três últimos anos. As localidades com $T$. infestans, consideradas Prioridade 1, continuaram com ciclo anual de trabalho. Aquelas em que as pesquisas de rotina detectaram a presença de $T$. sordida ou P. megistus, com percentagem de infestação maior do que $5 \%$ para o intradomicílio e/ou $10 \%$ para o peridomicílio, foram consideradas Prioridade 2, com ciclo de tratamento bienal. As demais localidades foram enquadradas na Prioridade 3, com ciclo de trabalho quadrienal. A proposta buscava racionalizar custos e operações de campo e aprimorar o trabalho, concentrando as ações de pesquisa em poucas localidades ainda com T. infestans, ou com índices de infestação maiores pelas outras duas espécies, dando-se especial atenção às notificações de focos ${ }^{16}$. A infestação residual por T. infestans era um fato concreto, uma vez que 29 localidades, todas situadas na região de Sorocaba, compunham a área de Prioridade 1. Este número representava 0,5\% do total das 839.807 localidades que formavam a área endêmica paulista. Avaliação referente ao período de 1984 a 1989 indicou alterações na proposta de vigilância ${ }^{16}$. Índices de infestação maiores que zero foram observados em $24 \%$ das localidades da antiga área endêmica, restritas a uma pequena área do estado. Quanto aos triatomíneos, houve predomínio de T. sordida $(84,4 \%)$ com índices de infecção por T. cruzi de $1 \%$, seguido de P. megistus (17,5\%), com $9 \%$ de infectados e $T$. infestans, com $0,1 \%$ do total dos coletados, distribuído de forma isolada e dispersa, sem infecção por T. cruzi, perfazendo 195 exemplares. Ficou comprovada a participação da população na vigilância entomológica, 
sendo constatado que $78 \%$ dos exemplares de triatomíneos coletados no intradomicílio procederam de notificação, enquanto $92 \%$ dos focos peridomiciliares foram detectados nas pesquisas realizadas por equipes da SUCEN. O BHC, que até então vinha sendo utilizado no controle, foi substituído pela deltametrina (piretroide sintético), utilizada na formulação de suspensão concentrada a $5 \%$ e, posteriormente, por alphacipermetrina $250 \mathrm{mg} / \mathrm{m}^{2} \mathrm{com}$ indicação de revisão de avaliação do controle químico após 60 a 90 dias da borrifação.

Antes do final do período, as últimas localidades infestadas por T. infestans haviam deixado de ser classificadas como P1 e, em consequência, a campanha contra o principal vetor da doença de Chagas, no Estado de São Paulo, chegava ao fim ${ }^{9,16}$.

Nova reformulação no Programa de Controle foi implantada em 1990, agora sem a presença de infestação por T. infestans, as localidades passaram a ter a seguinte classificação: Prioridade 1: localidades com índice de infestação intradomiciliar maior ou igual a $5 \%$ e/ou peridomiciliar maior ou igual a $10 \%$ (pesquisa bienal); Prioridade 2: localidades com índice de infestação intradomiciliar menor do que 5\% e peridomiciliar menor do que $10 \%$ (pesquisa bienal em uma amostra de localidades) e Prioridade 3: localidades com índice zero de infestação, portanto, com suspensão da pesquisa de triatomíneos), confirmando o que havia sido preconizado por Rocha e Silva e cols ${ }^{8}$.

Na década de 90, foi detectada a presença de T. infestans no Estado de São Paulo em três episódios (Figura 1 e Tabela 3), sendo dois deles com exemplares isolados, em 1990 e 1994, transportados passivamente, oriundos de outros estados e o último, em 1999, com 108 exemplares localizados em ninhos de pássaros, foco este debelado, sem repercussões para a população local ${ }^{17}$. A eliminação de Triatoma infestans no Estado de São Paulo foi fato marcante, tendo sido pioneiro no controle desta espécie no Brasil ${ }^{9}$.

Atualmente, dentre as espécies coletadas com maior freqüência e densidade, destacam-se T. sordida e Rhodnius neglectus dispersos na região do planalto paulista; P. megistus, encontrado nas regiões de Mata Atlântica e na Encosta Ocidental da Mantiqueira, em municípios que fazem divisa com o Estado de Minas Gerais e T. tibiamaculata, encontrado na região do litoral paulista, área de Mata Atlântica. No período de 1990 a 2001, foram capturados 93.142 exemplares de triatomíneos, dentre os quais $83,9 \%$ pertencentes à espécie T. sordida. A distribuição das espécies nos 645 municípios existentes no estado indica a presença de T. sordida em 48,5\% dos municípios; $R$. neglectus em 43,2\%; P. megistus em 35\% e T. tibiamaculata em 3,1\% deles. Quanto à infecção natural destas espécies, $P$. megistus permanece com os maiores índices de infecção, a partir da década de 1980, com valores no patamar de 10\%; T. sordida em patamares inferiores a $1 \%$ e R. neglectus com valores próximos a $1 \%$. A sorologia empregada para verificação de infecção chagásica tem detectado indivíduos positivos com aquisição em períodos anteriores à interrupção da transmissão vetorial no estado, portanto indivíduos com idades superiores a 35 anos, ou provenientes de outros Estados no Brasil onde a transmissão foi interrompida mais tardiamente ${ }^{18,19}$.

A partir do ano de 2003 o Programa suspendeu definitivamente a busca ativa de triatomíneos por parte da SUCEN, restringindo a vigilância à notificação de triatomíneos pela população ${ }^{20}$. A vigilância tem como norma incentivar notificações de insetos suspeitos, feitas pela população e recebê-las por intermédio das Unidades Básicas de Saúde, das Escolas ou diretamente dos Serviços Regionais da SUCEN. O encaminhamento de triatomíneos desencadeia uma pesquisa integral na casa notificante, com direcionamento para os locais de abrigo de animais utilizados como fonte alimentar pelos triatomíneos, uma atividade designada atendimento à notificação, dentro de um prazo máximo de 60 dias, a contar do recebimento. Independentemente do resultado deste atendimento, e sempre que as condições o permitem, são pesquisados integralmente os domicílios situados dentro de raio de 100 metros ao redor da moradia notificante. Nos domicílios em que foram encontrados triatomíneos, é realizado controle químico por meio de inseticidas da classe dos piretroides, sendo programada uma revisão deste controle químico, 60 a 90 dias após sua execução. Os triatomíneos coletados têm sido identificados e examinados quanto à positividade para tripanosomatídeos e por meio de reação de precipitação é identificada a origem do sangue ingerido, independentemente do local onde se deu a captura do inseto: domicílio ou peridomicílio. A prova é feita com a utilização de antissoros humano, marsupial, roedor, canídeo, felídeo e de aves. Exames sorológicos de moradores de unidades domiciliares com presença de colônias intradomiciliares de triatomíneos infectados por T. cruzi são realizados, utilizando-se as técnicas de imunofluorescência indireta (RIFI) e imunoensaio enzimático (ELISA). Em domicílios com identificação de soropositivos, é realizada a investigação epidemiológica familiar com especial atenção àqueles nascidos no Estado de São Paulo, com idades iguais ou inferiores a 35 anos $^{19}$.

o componente educativo passou a ter um peso importante para a sustentabilidade da vigilância entomológica e têm levado em consideração as premissas estabelecidas pelo Sistema Único de Saúde sobre as competências das três esferas de governo nas ações de epidemiologia e controle de doenças (Portaria 3252).

As atividades de vigilância e controle vetorial, assessoria técnica e capacitação dos recursos humanos permaneceram sob responsabilidade do estado, com utilização da rede de ensino e de saúde municipais para o desenvolvimento das ações educativas e de orientação à população, além da recepção dos insetos suspeitos.

Os resultados obtidos nos últimos 20 anos demonstram boa cobertura do programa constatando-se média de 2.350 notificações/ano, com valores variando de 788 a 4342 . O atendimento a essas notificações, representado pelas pesquisas realizadas nos domicílios notificantes, revelou o encontro de foco em $26,7 \%$ (Tabela 4). As regiões noroeste e sul são aquelas com maior concentração de notificações, em distribuição realizada segundo gradiente por município nos últimos 4 anos (Figura 2).

O cálculo do custo atual do Programa considera os dados da Tabela 4, no que diz respeito ao número de imóveis trabalhados em pesquisa e borrifação. A Tabela 5 apresenta o custo dos diversos itens que compõem a atividade, considerando-se para as atividades de campo os rendimentos médios para atendimento com pesquisa negativa e com pesquisa positiva, ou seja, acompanhada de borrifação. 
Os valores apresentados representam uma das faces do atendimento uma vez que sua qualidade, ou seja, o cuidado no trabalho, a atenção e os esclarecimentos prestados ao notificante são valores a considerar, embora de difícil mensuração.

Em síntese, os valores investidos nas diversas fases do Programa revalidam os resultados discutidos anteriormente, como pode ser observado na Tabela 6. De fato, as ações nas primeiras duas fases tiveram uma disponibilização discreta de recursos, quando comparado ao salto do investimento na fase do Arrastão, quando foram absorvidos os recursos excedentes da Campanha de Erradicação da Malária, cuja situação se apresentava bastante avançada, com ausência de

TABELA 4 - Notificações de triatomíneos atendidas e resultado dos atendimentos. Estado de São Paulo, 1984 a 2007.

\begin{tabular}{|c|c|c|c|c|c|}
\hline \multirow[b]{3}{*}{ Ano } & \multirow{3}{*}{$\begin{array}{c}\text { Notificações } \\
\text { atendidas }\end{array}$} & \multicolumn{4}{|c|}{ Resultado dos atendimentos } \\
\hline & & \multicolumn{2}{|c|}{ positivos } & \multicolumn{2}{|c|}{ negativos } \\
\hline & & $\mathrm{n}^{\circ}$ & $\%$ & $\mathrm{n}^{\circ}$ & $\%$ \\
\hline 1984 & 2.958 & 291 & 9,8 & 2.667 & 90,2 \\
\hline 1985 & 4.342 & 1.111 & 25,6 & 3.231 & 74,4 \\
\hline 1986 & 4.245 & 1.149 & 27,1 & 3.096 & 72,9 \\
\hline 1987 & 3.824 & 954 & 25,0 & 2.870 & 75,0 \\
\hline 1988 & 3.612 & 1.004 & 27,8 & 2.608 & 72,2 \\
\hline 1989 & 3.447 & 1.165 & 33,8 & 2.282 & 66,2 \\
\hline 1990 & 2.868 & 710 & 24,7 & 2.158 & 75,3 \\
\hline 1991 & 2.567 & 616 & 24,0 & 1.951 & 76,0 \\
\hline 1992 & 2.536 & 728 & 28,7 & 1.808 & 71,3 \\
\hline 1993 & 2.071 & 497 & 24,0 & 1.574 & 76,0 \\
\hline 1994 & 2.298 & 722 & 31,4 & 1.576 & 68,6 \\
\hline 1995 & 1.575 & 783 & 49,7 & 792 & 50,3 \\
\hline 1996 & 1.892 & 642 & 33,9 & 1.250 & 66,1 \\
\hline 1997 & 2.067 & 497 & 24,0 & 1.570 & 76,0 \\
\hline 1998 & 1.352 & 360 & 26,6 & 992 & 73,4 \\
\hline 1999 & 1.221 & 307 & 24,2 & 914 & 74,8 \\
\hline 2000 & 1.318 & 310 & 23,5 & 1.008 & 76,5 \\
\hline 2001 & 1.375 & 383 & 27,8 & 992 & 72,2 \\
\hline 2002 & 788 & 221 & 28,1 & 567 & 71,9 \\
\hline 2003 & 1.209 & 311 & 25,8 & 898 & 74,2 \\
\hline 2004 & 1.490 & 492 & 33,0 & 998 & 66,9 \\
\hline 2005 & 3.441 & 923 & 26,8 & 2.518 & 73,2 \\
\hline 2006 & 2.250 & 496 & 22,0 & 1.754 & 78,0 \\
\hline 2007 & 1.663 & 403 & 24,2 & 1.260 & 75,8 \\
\hline Total & 56.409 & 15.075 & 26,7 & 41.334 & 73,3 \\
\hline
\end{tabular}

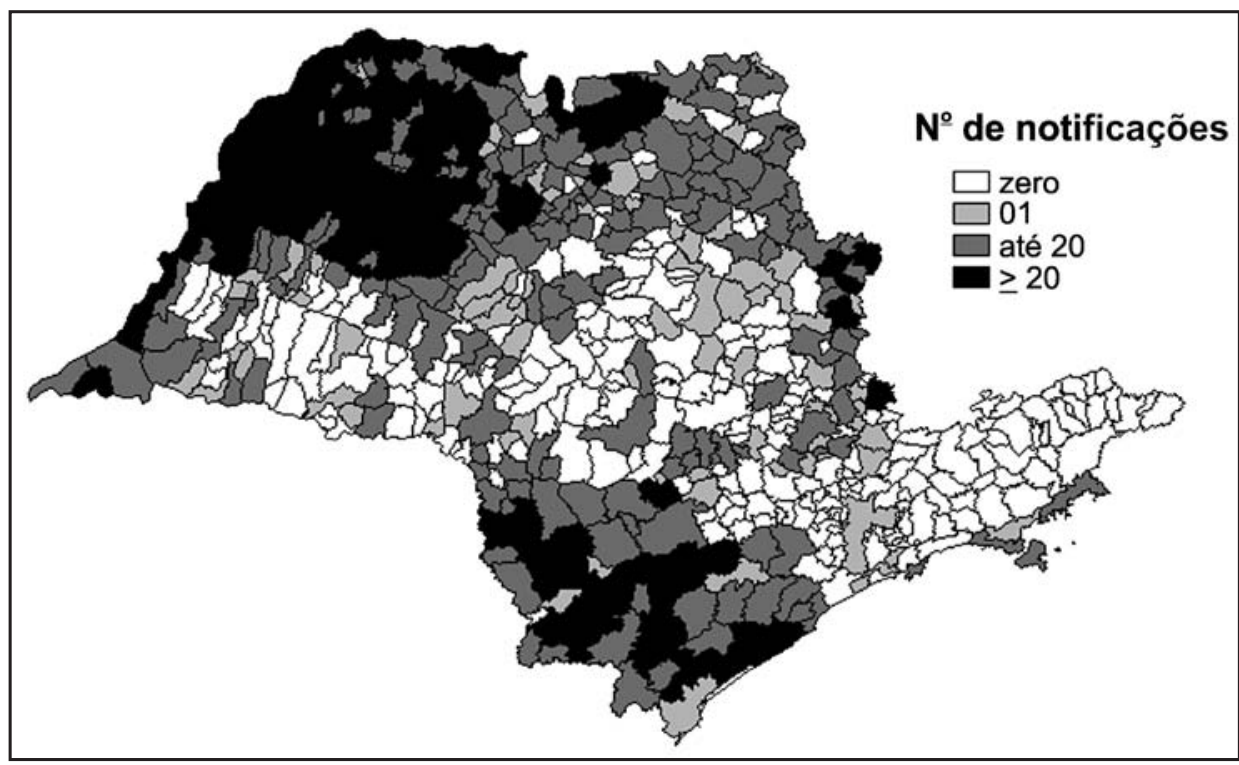

FIGURA 2 - Distribuição das notificações de triatomíneos, no Estado de São Paulo, 2004 a 2007. 
TABELA 5 - Dimensionamento do custo anual para as atividades de vigilância entomológica da doença de Chagas, no Estado de São Paulo.

\begin{tabular}{lrrrr}
\hline Itens & Investimento & Custeio & Total & US\$ \\
\hline I. Pessoal e reflexos & & $914.851,12$ & $914.851,12$ & $561.258,36$ \\
II. Diárias de campo & $171.219,84$ & $171.219,84$ & $105.042,85$ \\
III. Equipamentos & $10.032,00$ & 880,43 & $10.912,43$ & $6.694,74$ \\
IV. EPIs & & $34.320,00$ & $34.320,00$ & $21.055,21$ \\
V. Uniforme & & $1.500,00$ & $1.500,00$ & 920,25 \\
VI. Transporte & $544.500,00$ & $6.580,43$ & $551.080,43$ & $338.086,15$ \\
VII. Insumos & & $4.284,00$ & $4.284,00$ & $2.628,22$ \\
VIII. Material de campo* & & $5.000,00$ & $5.000,00$ & $3.067,48$ \\
\hline Total & $554.532,00$ & $\mathbf{1 . 1 3 8 . 6 3 5 , 8 2}$ & $\mathbf{1 . 6 9 3 . 1 6 7 , 8 2}$ & $\mathbf{1 . 0 3 8 . 7 5 3 , 2 6}$ \\
\hline *in
\end{tabular}

*inclui material de pesquisa entomológica, impressos e mostruário.

TABELA 6 - Custo do Programa de Controle da doença de Chagas, no Estado de São Paulo segundo fases.

\begin{tabular}{|c|c|c|c|c|}
\hline Fase & Período & Custo & $\mathrm{R} \$$ a preço $2008^{\mathrm{d}}$ & US\$ $\$^{e}$ \\
\hline Intermitente & 1953 a 1958 & $\mathrm{CR} \$ 5.382 .127,25^{\mathrm{a}}$ & $3.149 .000,00$ & $1.933 .000,00$ \\
\hline Trabalho intensificado & 1959 a 1964 & $\mathrm{CR} \$ 5.542 .066,75^{\mathrm{b}}$ & $2.570 .000,00$ & $1.577 .000,00$ \\
\hline Arrastão & 1965 a 1967 & $\operatorname{Cr} \$ 69.631 .962,66^{\mathrm{b}}$ & $32.289 .000,00$ & $19.820 .000,00$ \\
\hline Seletivo & 1968 a 1972 & $\mathrm{Cr} \$ 37.084 .446,12^{\mathrm{b}}$ & $17.196 .000,00$ & $10.600 .000,00$ \\
\hline Prioridades & 1973 a 1983 & $\operatorname{Cr} \$ 47.969 .966,00^{\mathrm{b}}$ & $22.000 .000,00$ & $13.502 .000,00$ \\
\hline Vigilância entomológica & 1984 aos dias atuais & $\mathrm{R} \$ 1.693 .167,82^{\mathrm{c}}$ & $1.693 .167,82$ & $1.038 .753,26$ \\
\hline \multicolumn{5}{|c|}{${ }^{2}$ Dotação orcamentária a preço de 1951 (Buralli, 1985). } \\
\hline \multicolumn{5}{|c|}{${ }^{b}$ Despesas de custeio realizadas a preço de 1978 (Rocha e Silva et al, 1979). } \\
\hline \multicolumn{5}{|c|}{ 'Despesas de custeio realizadas a preco de 2008.} \\
\hline \multicolumn{5}{|c|}{ dindice Geral de Precos DI-FGV. Valores arredondados. } \\
\hline \multicolumn{5}{|c|}{ eCotação US\$ em 30/05/2008 } \\
\hline \multicolumn{5}{|l|}{ Fonte: www.bcb.gov.br } \\
\hline
\end{tabular}

transmissão em grandes áreas do estado, recém saídas da chamada fase de Ataque. Também se observa a redução do custo para $50 \%$ quando da implantação do método seletivo, que permitiu o controle definitivo das áreas remanescentes de transmissão pelo T. infestans, com cobertura integral da área endêmica com borrifação precedida da pesquisa positiva. 0 mesmo pode ser constatado para a fase de prioridades, que embora preconizado um espaçamento nas pesquisas devido à classificação dos municípios, na prática, dada a existência de saldo operacional, muitos municípios seguiram com pesquisa anual. O custo da vigilância aqui apresentado considerou apenas os atendimentos às notificações. Embora no período de 1984 a 2001 tenham sido levadas a cabo pesquisas programadas em localidades, direcionadas para áreas de maior infestação, julgou-se oportuno demonstrar o custo da vigilância na ausência de busca ativa. Registra-se que enquanto no final da década de 1970 os custos com Chagas representavam cerca de $50 \%$ do total de despesas correntes da Autarquia, em 1995 este percentual fica restrito a 3\%.

\section{DISCUSSÃO}

As ações de controle vetorial desenvolvidas em São Paulo, com ênfase no Triatoma infestans lograram êxito no sentido de interromper a transmissão do Trypanosoma cruzi ao homem e foram devidamente documentadas pelos tropicalistas no final da década de 70 e início dos anos de $1980^{1,7,9,15,21}$. No contexto da doença, o processo migratório foi importante elemento para a dispersão da mesma, demonstrando um relacionamento com o tipo de colonização que ocorreu no interior paulista, interligados a expansão da fronteira agrícola, com o povoamento das áreas florestadas, virgens ou com população escassa ${ }^{1,7}$. Até o final da década de 30 , a cafeicultura subordinou-se a uma série de processos sociais, enquanto fator de estabilidade econômica, política e social. A expansão da doença e do seu principal vetor para as dimensões que atingiu o Estado de São Paulo teve seus limites definidos no espaço e no tempo, dentro de um processo social, que não ocorre nos dias atuais ${ }^{7}$. As análises da época, dos indicadores entomológicos e sorológicos conduziam ao consenso sobre o marco do controle em São Paulo. No entanto, tais análises indicavam, ao lado do excelente trabalho de fôlego conduzido pela Secretaria de Saúde de São Paulo, a contribuição concomitante do desenvolvimento econômico e social, ocorrido durante todos os longos anos da luta antivetorial, como a ocupação do espaço rural, o aumento da renda per capita no meio rural, a melhoria da habitação, a elevação do nível de escolaridade da população e o êxodo rural. As atividades de controle do vetor foram determinadas por injunções políticas, econômicas e técnicocientíficas, atrelados ao desenvolvimento verificado na zona rural paulista. $O$ peso relativo de cada um desses fatores foi destacado como de difícil avaliação, porém compunham um sistema de relações que contribuiu para o desaparecimento da endemia ${ }^{1}$. 
Durante a década de 50, o ciclo da terra virgem em São Paulo cessou, interrompendo um processo de desbravamento. As frentes pioneiras se deslocaram para os Estados do Paraná, Mato Grosso do Sul e Goiás. A taxa de urbanização sofreu uma aceleração do ritmo e de intensidade, resultado da crescente industrialização nucleada na Cidade de São Paulo e seu entorno, associada à crescente capitalização do campo e progressiva mecanização. Estes fatores culminaram em um importante decréscimo no número de domicílios rurais, refletindo diretamente no número de exemplares de triatomíneos coletados e infectados, resultado da não disponibilidade de ecótopos e de fonte de infecção. Ao final dos anos 1960, falava-se em interrupção da transmissão natural da doença de Chagas ${ }^{1,7}$.

O Programa de Controle da Doença de Chagas encontra-se hoje numa fase avançada mantendo-se a vigilância entomológica. As informações mais importantes são: - o encontro esporádico de exemplares de Triatoma infestans capturados, vindos de transporte passivo, e o não encontro de positividade para Trypanosoma cruzi nos mesmos; - a predominância da espécie Triatoma sordida, comprovadamente com baixa capacidade de transmissão, preferência alimentar por sangue de aves e baixa positividade para T. cruzi; - ocorrência de Panstrongylus megistus, espécie com maior capacidade de domiciliação, em áreas restritas do estado e - o encontro de sororreagentes para T. cruzi com idade superior a 35 anos. Atualmente, têm sido pouco expressivos os sinais de domiciliação das espécies secundárias, de acordo com os dados disponíveis.

A eliminação do T. infestans promoveu um olhar sobre as espécies secundárias. Os estudos clássicos realizados por Forattini e cols com a participação do Centro de Pesquisa em Doença de Chagas da SUCEN em Mogi Guaçu, na década de 1970, na série Aspectos Ecológicos da Tripanossomíase Americana, são, até hoje, de fundamental importância para compreensão dos mecanismos comportamentais das espécies, contribuindo enormemente para direcionamento e normatização das estratégias de controle. Aliás, desde 60 este Centro vem desenvolvendo pesquisas na área de controle dos triatomíneos, cujos resultados colaboram com as atividades do programa.

Encerrada com sucesso a campanha contra T. infestans, principal transmissor da doença de Chagas no estado, as atividades de controle não terminaram. Seu prosseguimento se justifica:

a) pela possível reintrodução de T. infestans, por transporte passivo e conseqüente necessidade da vigilância; b) pela continuação das atividades de controle dirigidas à presença, nas UDs dos exemplares de $T$. sordida ou $P$. megistus, espécies ditas secundárias; c) pela invasão das UDs por exemplares de outras espécies de triatomíneos, tais como: Rhodnius neglectus, no planalto, e Triatoma tibiamaculata e P. megistus, no litoral. d) pelo atendimento às notificações de triatomíneos e estímulo a continuidade dessa prática, de grande utilidade na vigilância.

Incentivos às atividades de vigilância entomológica são de grande valor na medida em que a mesma avança e outros problemas epidemiológicos passam a exigir maior atenção. Os Programas de Saúde da Família e do Agente Comunitário de Saúde, particularmente no meio rural de áreas sensíveis ao problema, devem incluir ações que sensibilizem os moradores para a possível presença dos triatomíneos nas UDs e nos procedimentos de captura e encaminhamento dos exemplares para a unidade sanitária mais próxima. 0 mesmo deverá ocorrer no processo educativo junto às escolas ${ }^{22}$.

Em novembro de 1995, esteve no Brasil uma Comissão Internacional de Avaliação, patrocinada pela Organização Pan-americana de Saúde (OPAS), que emitiu, entre outros, o seguinte parecer: o T. infestans foi eliminado do Estado de São Paulo 9 . Os dados mais recentes publicados pela SUCEN ratificam o parecer acima e confirmam a inexistência da veiculação do T. cruzi ao homem através dos triatomíneos nas moradias do Estado de São Paulo ${ }^{19}$. De fato, todos os indicadores entomológicos e sorológicos evidenciam que a transmissão foi interrompida há mais de 30 anos. A maioria dos casos sororreagentes detectados pelo Programa de Controle na década de 90 estava associada à aquisição da infecção em áreas sabidamente endêmicas para Doença de Chagas no Estado, até fins dos anos de 1960, ou fora deste estado, onde o controle da transmissão tardou a ocorrer ${ }^{18}$.

Há confirmação da persistência de Triatoma sordida, principalmente no peridomicílio com baixos índices de infecção natural. Os resultados corroboram com o fato de que não ocorre veiculação do T. cruzi ao homem por triatomíneos domiciliados, ou seja, a transmissão através das fezes dos triatomíneos que ao picarem os vertebrados, defecam após o repasto eliminando formas infectantes. Outra evidência que confirma o exposto acima está baseada na realização do inquérito de soroprevalência, que ocorreu em todo o país, dirigido a crianças do grupo etário de 0 a 5 anos. No Estado de São Paulo, após processadas aproximadamente 4.725 amostras, originárias de diferentes áreas, abrangendo 238 municípios, não foi confirmado nenhum caso. No entanto, estes resultados não interromperão os investimentos na vigilância entomológica da doença aplicada pela SUCEN e a vigilância epidemiológica terá papel fundamental no controle da mesma com vistas à rápida detecção de surtos episódicos que possam ocorrer.

No atual contexto do controle da transmissão da doença de Chagas, no Estado de São Paulo, a detecção da forma aguda da doença deve tornar-se cada vez mais rara, e o mecanismo de transmissão deve ser exaustivamente investigado, como aconteceu com o caso confirmado na Baixada Santista em 1995, em que o paciente foi a óbito, com história de excursão em ambiente silvestre localizado entre os municípios de Itanhaém e Peruíbe, região de Mata Atlântica, no Vale do Ribeira, com pernoite em domicílios livres da presença de triatomíneos ${ }^{23}$ e o recente caso de óbito em criança que teve a doença confirmada por necropsia no município de Itaporanga, Região de Botucatu, em cuja residência não foi constatada a presença de foco de triatomíneos ${ }^{24}$.

Uma vez que o agente etiológico, protozoário flagelado, possui como reservatórios, além do homem, mamíferos silvestres e domiciliados que coabitam ou estão próximos do homem, em condições socioepidemiológicas que permitem a infecção humana, acidentes poderão ocorrer no manuseio desses animais, ou mesmo a ingestão de carne mal cozida, fato este já constatado em São Paulo ${ }^{25,26}$.

O modelo de vigilância desenvolvido que envolve a participação popular e os serviços locais de saúde e educação, permitem garantir sustentabilidade das ações, assegurando 
a detecção dos triatomíneos e monitorando situações que envolvam risco. Devem, ainda, ser implementadas ações que busquem orientar a população para a adequada modificação do ambiente peridomiciliar com vistas a dificultar o estabelecimento de colônias desses insetos, principalmente em áreas ocupadas por população originária de áreas urbanas que se instalam em ambiente rural. Fato este registrado pelo IBGE que na última década mostrou um acréscimo no número de domicílios rurais, devido àoportunidade de emprego em projetos turísticos, como pesqueiros e hotéis-fazenda, entre outros, sem conhecimento da epidemiologia da doença de Chagas.

Neste contexto, cada vez mais as ações de educação em saúde devem nortear os princípios do Sistema Único de Saúde de universalização, descentralização, integralidade e participação da comunidade, centrada na promoção da qualidade de vida. A proposta de vigilância instituída contribui para a melhor estruturação da SUCEN, como órgão de pesquisa, consolidando-a como referência na normatização e investigação operacional de métodos de controle de vetores de importância em saúde pública. Nesse novo contexto, é esperado que as estratégias de vigilância epidemiológica, adotadas pela SUCEN, mantenham resguardados os excelentes resultados até então alcançados.

\section{REFERÊNCIAS}

1. Silva LJ. A Evolução da doença de Chagas no Estado de São Paulo. [Tese de Doutorado] São Paulo, SP: Editora HUCITEC;1999.

2. Fonseca JAB, Passalacqua CSP, Lima AR, Oliveira AP. Lacerda JHM. Índices de infecção de triatomíneos no Estado de São Paulo. Arq Hig Saúde Públ 1952; 17:133-136.

3. Carini A.Considerações sobre a moléstia de Chagas. Palestra realizada no Instituto Biológico em 8 de março de 1940. Arq Biol 1940; 22:77-84.

4. Pessoa SB, Lima F, Santos JA. Sobre encontro de mais de sete casos de moléstia de Chagas no município de Itaporanga (Estado de São Paulo). Rev Médica 1942; 26:11-20.

5. Pessoa SB, Villela F. Primeiro caso de moléstia de Chagas (aguda) no município de Araçatuba, Estado de São Paulo. Rev Paulista de Medicina 1943; 22:240.

6. Pessoa SB. Coutinho JO. Forma aguda da moléstia de Chagas no município de Assis (Estado de São Paulo). Rev Clin São Paulo 1949; 25:89-90.

7. Buralli GM. Estudo do controle dos triatomíneos domiciliados no Estado de São Paulo. [dissertação]. São Paulo, SP: Faculdade de Saúde Pública da Universidade de São Paulo,1985.

8. Silva EOR, Guarita OF, Ishihata GK. Doença de Chagas: atividades de controle dos transmissores no Estado de São Paulo. Rev Bras Malariol D Trop 1979; 31:99-119.

9. EOR, Wanderley DMV, Rodrigues VLCC. Triatoma infestans: importância, controle e eliminação da espécie no Estado de São Paulo, Brasil. Rev Soc Bras Med Trop 1998; 31:73-88.

10. Freitas JLP. Importância do expurgo seletivo para a profilaxia da moléstia de Chagas pelo combate aos triatomíneos. Arq Hig Saude Públ 1963; 28:212-272.
11. Corrêa RR, Ferreira AO. Distribuição geográfica, habitats e infecção do T. sordida no Estado de São Paulo. Rev Inst Med Trop São Paulo 1959; 1: 207-213.

12. Coutinho JO. Contribuição ao estudo da epidemiologia da doença de Chagas. Arq Hig Saude Publ 27:317-333, 1962.

13. Silva EOR, Dias Jr J, Guarita OF . Suspensão do rociado no combate ao Triatoma infestans em área do Estado de São Paulo, Brasil. Rev Saude Publ 1969; 3:173-8.

14. Silva, EOR, Andrade JCR, Rodrigues, VLCC. Investigação de Foco, uma das atividades das campanhas de controle dos transmissores da Tripanossomíase Americana. Rev Saude Publ 1978; 12:425-431.

15. Souza AG, Wanderley DMV, Buralli GM, Andrade JCR. Consolidation of the control of Chagas' Vectors in the state of São Paulo. Mem Inst Oswaldo Cruz 1984; 79:125-132.

16. Wanderley DMV. Perspectivas de controle da doença de Chagas no Estado de São Paulo. [Tese de doutorado], São Paulo, SP: Faculdade de Saúde Pública, USP, 1994.

17. Leite OF, Alves MJCP, Souza SSL, Mayo RC, Andrade VR, Souza CE, Rangel O, Oliveira SS, Lima VLC, Rdrigues VLCC, Carvalho ME, Casanova C, Wanderley DMV. Triatoma infestans em área de vigilância para doença de Chagas, Estado de São Paulo, Brasil. Rev Soc Bras Med Trop 2001; 34:43743.

18. Carvalho ME, Silva RA, Rodrigues VLCC, Oliveira CD. Programa de Controle da Doença de Chagas no Estado de São Paulo: sorologia de moradores como parte de investigação de unidades domiciliares com presença de triatomíneos vetores na década de 1990. Cad Saude Publica 2002;18:16951703.

19. Wanderley, DMV, Silva RA, Carvalho ME, Barbosa GL. Doença de Chagas: a vigilância entomológica no Estado de São Paulo. Bol Epid Paul 2007; 4:10-14.

20. Secretaria de Estado da Saúde do Estado de São Paulo (SES). Relatório do Grupo de Trabalho, Revisão do Programa de Controle da Doença de Chagas, São Paulo. São Paulo: Superintendência de Controle de Endemias; 2002.

21. Caldas Jr AL. Epidemiologia e controle da doença de Chagas. Relação com a estrutura agrária na Região de Sorocaba, SP. [dissertação] São Paulo, SP: Faculdade de Medicina da Universidade de São Paulo; 1980.

22. Silva RA, Wanderley DMV, Domingos MF, Yasumaro S, Scandar SAS, Pauliquévis-Junior C, Sampaio SMP, Takaku L, Rodrigues VLCC. Doença de Chagas: notificação de triatomíneos no Estado de São Paulo na década de 1990. Rev Soc Bras Med Trop 2006; 39: 488-494.

23. Ciaravolo RMC, Domingos MF, Wanderley DMV, Gerbi LJ, Chiefi PP, Peres BA, Umezawa Es. Autochthonous acute Chagas' disease in São Paulo State, Brazil: Epidemiological Aspects. Rev Inst Med Trop São Paulo 1997; 39: 171-174.

24. Wanderley DMV, Tatto E, Yassuda MAS, Carvalgho ME, Leite RM, Santos SO, Diaz SY, Rodrigues VLCC. Caso agudo de doença de Chagas no Estado de São Paulo. Investigação Preliminar. XXII Reunião de Pesquisa Aplicada em Doença de Chagas e X Reunião de Pesquisa em Leishmanioses, Programas e Resumos; 2006.

25. Forattini OP, Rocha e Silva EO, Barata JMS, Boainain.E. Nota sobre caso autóctone de tripanossomíase americana no Litoral Sul do Estado de São Paulo, Brasil. Rev Saude Publ São Paulo 1980; 14:143-149.

26. Forattini OP, Rocha e Silva EO, Barata JMS, Boainain E. Nota sobre novo caso autóctone de tripanossomíase americana no Litoral Sul do Estado de São Paulo, Brasil. Rev Saude Publ 15:350-352. 